\title{
A Novel and Improved Firefly Algorithm Based on Two-order Oscillation
}

\author{
Yongbo Sui \\ Key Laboratory of Intelligent Computing \& Information Processing (Xiangtan University), \\ Ministry of Education, Xiangtan, 411105, China \\ E-mail: 201431111830@smail.xtu.edu.cn \\ Lingzhi $\mathrm{Yi}^{\dagger}$ and Wenxin $\mathrm{Yu}$ \\ Wind power equipment and power conversion 2011 Collaborative Innovation Center, Xiangtan, 411105, China \\ School of Information and Electrical Engineering Hunan University of Science and Technology, \\ Hunan Pro., Xiangtan, 411201, China \\ E-mail: \{ylzwyh@xtu.edu.cn, 13874894700@163.com \}
}

\begin{abstract}
Firefly algorithm is a new and efficient intelligent algorithm proposed by Dr. Xin-She Yang at Cambridge University. In this paper, the conventional firefly algorithm will be introduced and improved using two-order oscillation. And an improved firefly algorithm based on two-order oscillation is proposed. And this method will be deduced and analyzed for astringency. Six typical functions will be tested to prove performances. Global Max/Min values in six multimodal functions could be found accurately with proposed scheme. The experiment results show our proposed method has excellent performances than conventional one.
\end{abstract}

Index Terms-Firefly algorithm, Improvement, Twoorder Oscillation.

\section{INTRODUCTION}

Originated by bioluminescence, a new heuristic intelligence algorithm - firefly algorithm (FA), was developed by Dr. Xin-She Yang at Cambridge University in 2008 [1], with many similarities with other algorithms which are based on the so-called swarm intelligence, such as the famous Particle Swarm Optimization (PSO)[2], Artificial Bee Colony optimization (ABC), and Bacterial Foraging (BFA) algorithms, it is indeed much simpler both in concept and implementation. Due to its excellent performances, FA has been widely applied to many optimization fields, including optimization [3-4], data mining [5] and digital image processing [6] and so on.

However, one of the major drawbacks of FA is its premature convergence, especially when to handle problems with more local optima. Several attempts have been made to improve the performance of the basic FA to solve many optimization problems in different fields. Such as Luleseged and Hong Choon Ong et al. [7] modified the random movement of the brightest firefly that in some generations, when the current best position does not improve, may decreases its brightness. The proposed modification tries to improve the brightest firefly position generating the m-uniform random vectors and moves it in the direction of the best performance. In 2013, by replacing absorption coefficient and randomization parameter with modulo function of Gaussian distribution, Leandro dos Santos Coelhoa et al. proposed a method to avoid fireflies into local optimal locations, which has improved performance of firefly algorithm in chiller loading for energy conservation [8]. In 2014, Adil Baykasoglu et al.[9] has proposed an improved firefly algorithm with partial random restarts and an adaptive move procedure to solve dynamic multidimensional knapsack problems. For improvement of power quality in active power conditioner, Masoud Farhoodnea et al.[10] proposed a modified firefly algorithm with a tangent hyperbolic sigmoid function to update locations of fireflies; Similar to particle swarm optimization, global optimal location and individual optimal location ware drawn into firefly algorithm by $\mathrm{M}$. Gnana Sundari for adjustable DC sources in 2015 [11]. In 2016, M. Gnana Sundari et al.[12] has proposed a selfadaptive firefly algorithm through improving the random parameter $\alpha$ to optimize the angle of multilevel inverter with adjustable DC sources. Although those proposed methods could avoid fireflies into local optimal locations in a way, however, more parameters have to be set and controlled in proposed method, which increase complexity of algorithm. In addition, related mathematic derivations weren't given in most of literatures. So in this paper, an improved firefly algorithm will be explained with the stable theory of dynamic system and six typical functions will be used to verify performance. The simulative results indicate this modified firefly algorithm has excellent performance than standard one.

So the construction of the paper is: a vast literature survey has been conducted to review the state of the previous developed methods in section I; and in section II and section III, the basic theory of standard firefly algorithm is introduced by mathematical derivation. The stability of that will be proved. And the improved firefly algorithm will also be proved and deduced with twoorder oscillation. 


\section{FIREFLY ALGORITHM}

In this section, the basic theory of firefly algorithm will be introduced in detail.

In the process of optimization in firefly algorithm, the optimized result can be got via the progress of the position update, respective attraction and lightness of the fireflies [13-14]. The process of finding optimal solution is presented by the process of the fireflies searching the better locations in the solution space. Two important parameters are mentioned: the light intensity and the attractiveness. For simplicity, the following three idealized rules should be observed [15]:

1: All fireflies will be attracted to other regardless of their sex;

2: Attractiveness is proportional to their brightness, thus for any two flashing fireflies, the less bright one will move towards the brighter one.

3: The brightness of a firefly is affected or determined by the objective function.

In the simplest form, the light intensity $I$ varies according to the inverse square law:

$$
I=\frac{I_{o}}{r_{i j}^{2}}
$$

Where $I_{o}$ is the intensity at the source. In order to avoid the singularity at $r=0$ in the expression (1), the combined effect of both the inverse square law and absorption can be approximated as the following Gaussian form:

$$
I=I_{o} \times e^{-\gamma r_{i j}}
$$

Where $I_{o}$ is the original light intensity $(r=0)$ and $\gamma$ is the light absorption coefficient, $\gamma \in[0 . \infty]$.As a firefly's attractiveness is proportional to the light intensity seen by adjacent fireflies, we can now define the attractiveness $\beta$ of a firefly by:

$$
\beta=\beta_{o} \times e^{-\gamma r^{2}}
$$

Where $\beta_{o}$ is the attractiveness at $r=0$. The distance between any two firefly $i$ and $j$ at $x_{i}$ and $x_{j}$, respectively. The Cartesian distance is defined as:

$$
r_{i j}=\left|r_{i}-r_{j}\right|=\sqrt{\sum_{k=1}^{d}\left(x_{i, k}-x_{j, k}\right)^{2}}
$$

Where $x_{i, k}$ is the $k$-th component of the spatial coordinate $x_{i}$ of $i$-th firefly.

The movement of the $i$-th firefly is attracted to another more attractive (brighter) the $j$-th firefly is defined by:

$$
x_{i}(t+1)=x_{i}(t)+\beta\left(x_{j}(t)-x_{i}(t)\right)+\alpha\left(\operatorname{rand}-\frac{1}{2}\right)
$$

Where $x_{i}(t+1)$ represents the position of the firefly $i$ after $t+1$ th movement; On the equal mark right, the second term is due to the attraction, while the third term is randomization with $\alpha$ being the randomization parameter, $\alpha \in[0,1]$. For most problems, rand is random number which are drawn from a Gaussian distribution.

\section{The Design OF Firefly AlgOrITHM BASED ON TwO- ORDER OSCILLATION}

In this section, the improved firefly algorithm based on two-order oscillation will be described.

In the process of optimization, fireflies will move to brighter those, according to two important parameters: the light intensity and the attractiveness. The updated formula of locations is showed as following:

$$
x_{i}(t+1)=x_{i}(t)+\beta\left(x_{j}(t)-x_{i}(t)\right)+\alpha\left(\operatorname{rand}-\frac{1}{2}\right)
$$

Assuming that the $j$-th firefly with the $t$-th iteration is brighter than that $i$-th firefly, random is added to avoid fireflies into local optimal locations, it can be regarded as a bias in iterative systems. So the formula (6) could be rewrote as following:

$$
x_{i}(t+1)-x_{i}(t)=\beta\left(x_{j}(t)-x_{i}(t)\right)
$$

The above could be regarded as difference formula of Euler for $x_{i}(t)$ and $x_{j}(t)$. So, its differential formula is:

$$
\frac{d x_{i}(t)}{d t}=-\beta x_{i}(t)+\beta x_{j}(t)
$$

So it could be rewrote with Laplace transformation: $s x_{i}(s)+\beta x_{i}(s)=\beta x_{j}(s)$, and is also explained:

$$
\frac{x_{i}(s)}{x_{j}(s)}=\frac{\beta}{s+\beta}
$$

As is showed above, it could be regarded as a oneorder inertial system, and $x_{j}(s)$ and $x_{i}(s)$ are the import and export of that, respectively. Since the iteration increasing, the export $x_{i}(s)$ will converge towards to $x_{j}(s)$, which means the conventional firefly algorithm has convergence in a way. However, this method just with inertial convergence couldn't balance searching step and searching accuracy, which may lead fireflies into local optimal locations. Hence, the convergent method is 
replaced with two-order oscillation in our paper. The proposed method is showed as:

$$
x_{i}(t+1)=x_{i}(t)+\beta\left(x_{j}(t)-(1+\varphi) x_{i}(t)-\varphi x_{i}(t-1)\right)+\varepsilon
$$

Where $\varepsilon=\alpha($ Rand -0.5$) ; x_{i}(t-1)$ is location of the $i$ th firefly at $(t-1)$-th iteration. And $\varphi$ is defined as balanced coefficient. At former period of iterations, $\varphi$ could be selected in $\left(0, \frac{2 \sqrt{\beta}-1}{\beta}\right)$ for big searching step; and it could be set: $\left(\frac{2 \sqrt{\beta}-1}{\beta}, \frac{3(2 \sqrt{\beta}-1)}{2 \beta}\right)$ at later period of iteration.

\section{Derivation and Proof:}

In a similar way, the formula (5) can be rewrote:

$$
\begin{aligned}
& x_{i}(t+1)-x_{i}(t)-\left(x_{i}(t)-x_{i}(t-1)\right) \\
& =\beta\left(x_{j}(t)-(1+\varphi) x_{i}(t)-\varphi x_{i}(t-1)\right)-\left(x_{i}(t)-x_{i}(t-1)\right)
\end{aligned}
$$

So,

$$
\begin{aligned}
& x_{i}(t+1)-2 x_{i}(t)+x_{i}(t-1) \\
& =-\beta(\varphi+1) x_{i}(t)-x_{i}(t)+\varphi \beta x_{i}(t-1)+x_{i}(t-1)+\varphi x_{j}(t) \\
& =-\beta \varphi\left(x_{i}(t)-x_{i}(t-1)\right)-\left(x_{i}(t)-x_{i}(t-1)\right)-\beta x_{i}(t)+\beta x_{j}(t)
\end{aligned}
$$

So the differential formula of above is:

$$
\frac{d^{2} x_{i}(t)}{d t^{2}}=-(\beta \varphi+1) \frac{d x_{i}(t)}{d t}-\beta x_{i}(t)+\beta x_{j}(t)
$$

The Laplace transformation of that is:

$$
s^{2} x_{i}(s)+(\beta \varphi+1) s x_{i}(s)+\beta x_{i}(s)=\beta x_{j}(s)
$$

That is:

$$
\frac{x_{i}(s)}{x_{j}(s)}=\frac{\beta}{s^{2}+(\beta \varphi+1) s+\beta}
$$

The above formula could be regarded as a two-order Oscillational system.

So when $s^{2}+(\beta \varphi+1) s+\beta=0$, two solutions are:

$$
A_{1,2}=\frac{-(\beta \varphi+1) \pm \sqrt{(\beta \varphi+1)^{2}-4 \beta}}{2}
$$

For big searching step and fast convergent speed, the system could be underdamping state and be convergent. So, $(\beta \varphi+1)^{2}-4 \beta<0$ and $-(\beta \varphi+1)<0$, that is, the balanced coefficient could be selected in $\left(0, \frac{2 \sqrt{\beta}-1}{\beta}\right)$ at former period of iterations. At later period, this system should be in over damping state for small searching step and high precision: $(\beta \varphi+1)^{2}-4 \beta>0$ and $-(\beta \varphi+1)<0$. After numerous experiments, it can be set in $\left(\frac{2 \sqrt{\beta}-1}{\beta}, \frac{3(2 \sqrt{\beta}-1)}{2 \beta}\right)$.

\section{The Simulation AND EXPERIMENT}

In this section, the original firefly algorithm proposed by Xin-She Yang will be regarded as standard method to test with six different functions and compare with our improved firefly algorithm using MATLAB/Simulink. And we could explain and verify the feasibility and effectiveness of our proposed method.

\section{A. Six Tested Functions}

The efficiency and performance of the proposed method with the following six nonlinear functions (11-16) are evaluated:

$$
\begin{aligned}
f_{1}(x, y) & =e^{-(x-4)^{2}-(y-4)}+e^{-(x+4)^{2}-(y-4)^{2}} \\
& +2\left[e^{-x^{2}-y^{2}}+e^{-x^{2}-(y+4)^{2}}\right]
\end{aligned}
$$

Where $(x, y) \in[-5,5] \times[-5,5]$

$$
f_{2}(x, y)=\frac{\sin \left(\sqrt{x^{2}+y^{2}}\right)}{\sqrt{x^{2}+y^{2}}}+e^{\frac{\cos (2 \pi x)+\cos (2 \pi y)}{2}}-2.71289
$$

Where $(x, y) \in[-2,2] \times[-2,2]$

$$
f_{3}(x, y)=e^{-\sin ^{2} x-\cos ^{2} y}+2 e^{-\cos ^{2} x-\sin ^{2} y}
$$

Where $(x, y) \in[-2,2] \times[-2,2]$

$$
f_{4}(x, y)=0.5-\frac{\sin ^{2} \sqrt{x^{2}+y^{2}}-0.5}{\left(1+0.001\left(x^{2}+y^{2}\right)\right)^{2}}
$$

Where $(x, y) \in[-200,200] \times[-200,200]$

$$
f_{5}=\sum_{i=1}^{n}\left(x_{i}^{2}-10 \cos \left(2 \pi x_{i}\right)+10\right)
$$

Where $-5<x_{i}<5$.

$$
f_{6}=\sqrt{\sum_{i=1}^{n} x_{i}^{2}}
$$


Where $-4<x_{i}<4$.

Function $f_{1}$ is the four-peak function as illustrated in Fig.1(a), which has two local peaks with $f=1$ at $(-4,4)$ and $(4,4)$, and two global peaks with $f_{\max }=2$ at $(0,0)$ and $(0,-4)$. Function $f_{2}$ is showed in Fig.1(b), which has many local maximum and one global maximum $f_{1}=1$ at $(x, y) \rightarrow(0,0)$.Function $f_{3}$ has two local maximum and two global peaks with $f_{\max }=2.135$ at $(1.6,0)$ and $(-1.6,0)$, which is showed in Fig.1(c). Function $f_{4}$ is the Schaffer's function as illustrated in Fig. 1(d), which has infinite local maximum and one global maximum at $(0,0)$, and $f=1$. Opposing to $f_{2}, f_{5}$, named Rastrigin, has same innumerable local maximum and minimum points, and one global minimum point is $(0,0)$, that is, $f_{5 \min }=0$; The sixth functions is Sphere function, liking a bowl, has one global minimum location at $(0,0)$. When function lies this point, the global minimum value could be got, that is, $f_{6 \min }=0$, as is shown in Fig.1.(f).

\section{B. Discussion and Analysis}

Fig.2 has showed final locations of fireflies for different functions with stand and proposed method, and the relevant parameters are followed: In $f_{1} \sim f_{6}$, the population size of firefly and the max iterations are 12 and 50, respectively;Tab.1 has presented optimal locations found by fireflies. As we can see from Fig. 2(a) and Fig. 2(b), all fireflies have moved to two global peaks oppose to six fireflies got in local maximum value, although the precision is similar to standard method. In function 2, all bodies have come to a local optimal location, unfortunately. However, the global optimal

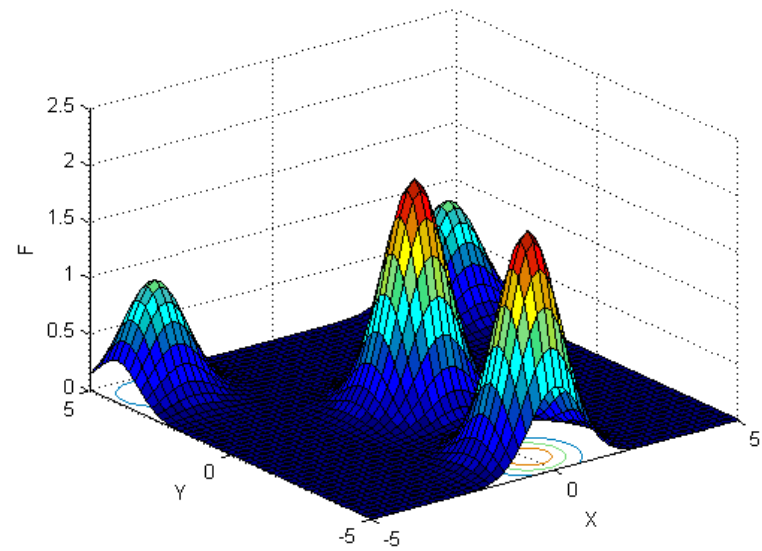

(a) $f_{1}$ location can be easily found after several iterations. As for function 3, only one global point could be found and others have moved to local optimal point, mistakenly. Compared to it, our proposed method still has excellent ability to recognize and find two global max locations. Likewise, the optimal point could be found facing to function 4 with infinite local peaks. In Fig.2(i) and Fig.2(j), While the optimal point couldn't be found in spite of several iterations, that point can be easily identified, that is, 0.1466 , opposing to standard mothod with 5.0538. As we can see from Fig.1(k) and Fig.1(1), almost of all fireflies have moved to the only minimum location, no matter standard method or proposed that. Compared to 0.098 of former one, however, the latter minimum value 0.012 is more close to actual minimum value $f_{6 \min }=0$.

\section{CONCLUSION}

This paper has focused on an improved firefly algorithm based on two-order oscillation. Mathematical deduction of standard firefly algorithm indicates it has convergence performance in a way. A firefly will move to more lighter one with one order inertia process, which may lead to premature convergence, that is, local optimal locations. So the update method of one-order inertia is replaced with two-order oscillation, and range of balanced coefficient has been given to balance searching step and searching accuracy. The simulation results show that proposed method realizes more excellent performance to prevent individual into local optimal locations and obtain high accuracy than conventional method.

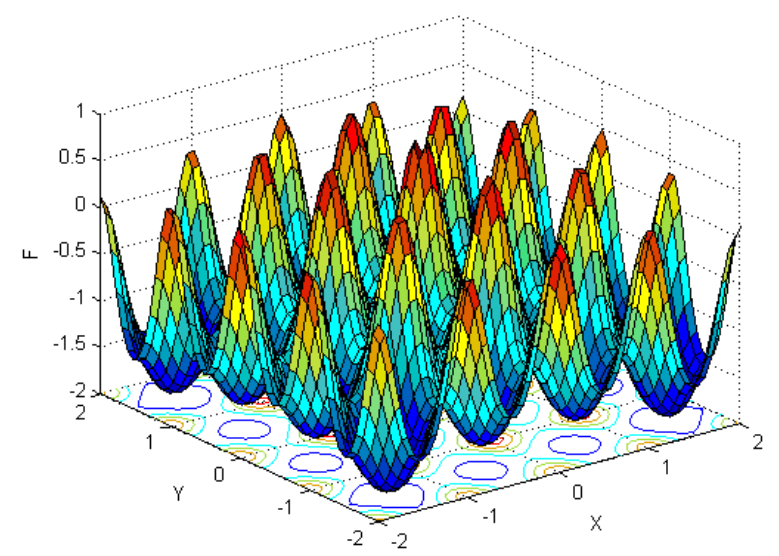

(b) $f_{2}$ 


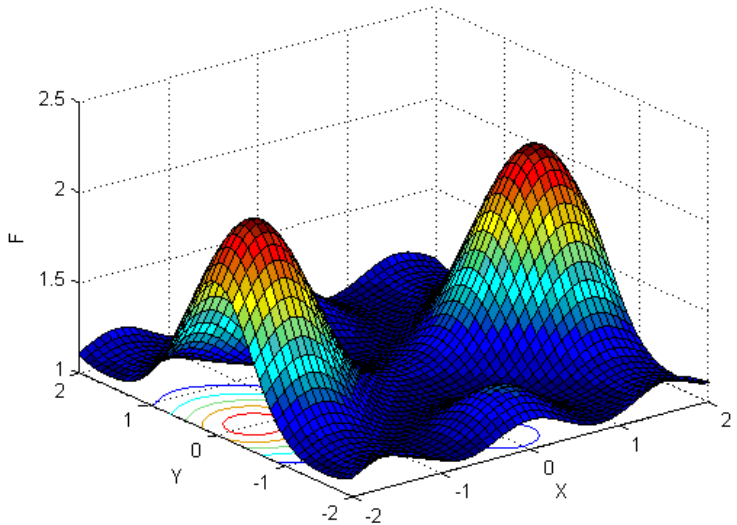

(c) $f_{3}$

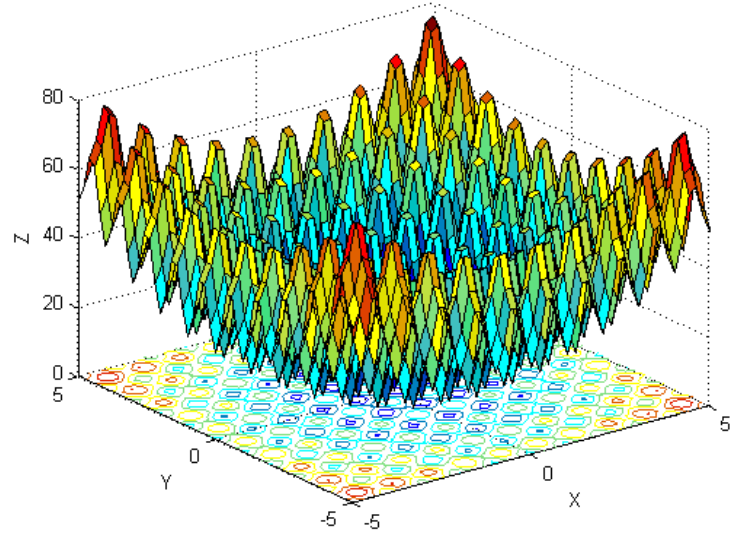

(e) $f_{5}$

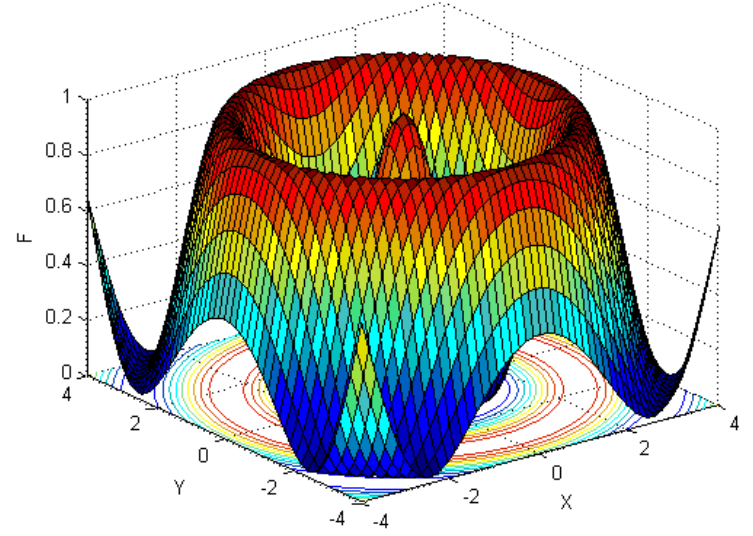

(d) $f_{4}$

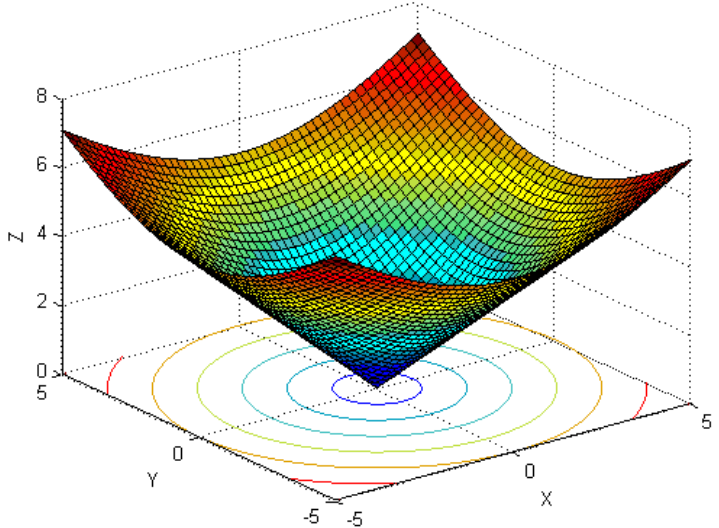

(f) $f_{6}$

Fig.1. Six Tested Functions

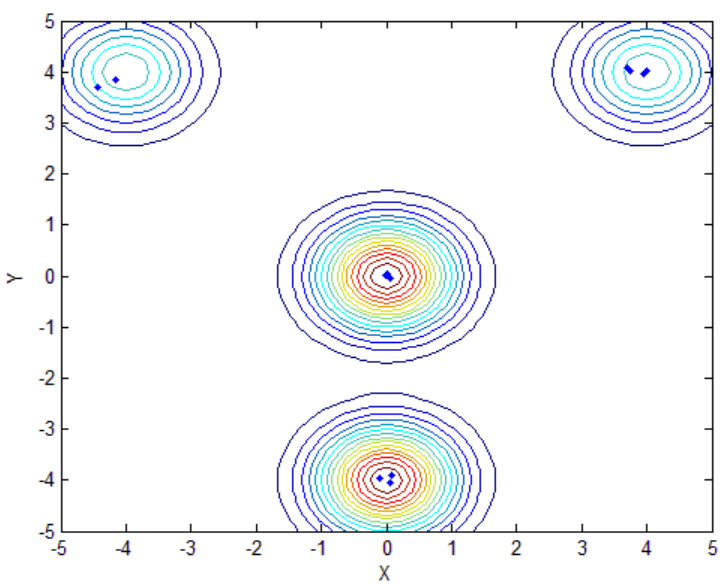

(a) Locations of fireflies for $f_{1}$ with standard metho

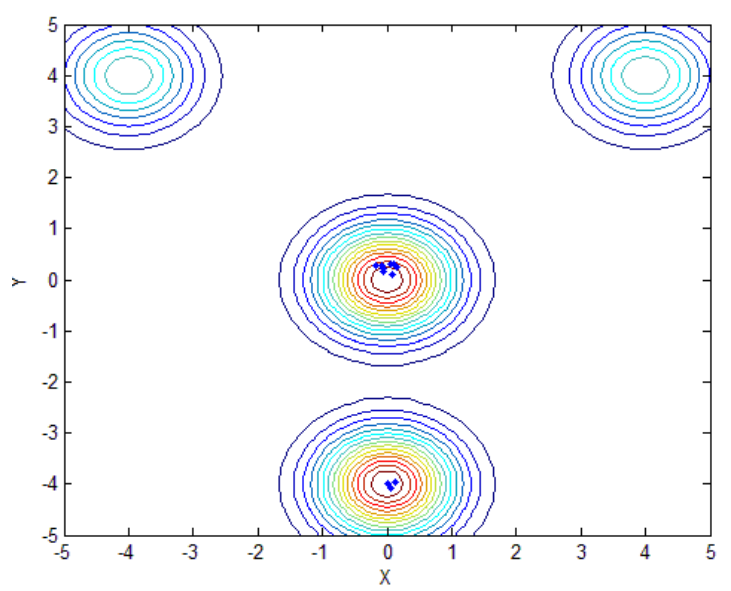

(b) Locations of fireflies for $f_{1}$ with proposed method 


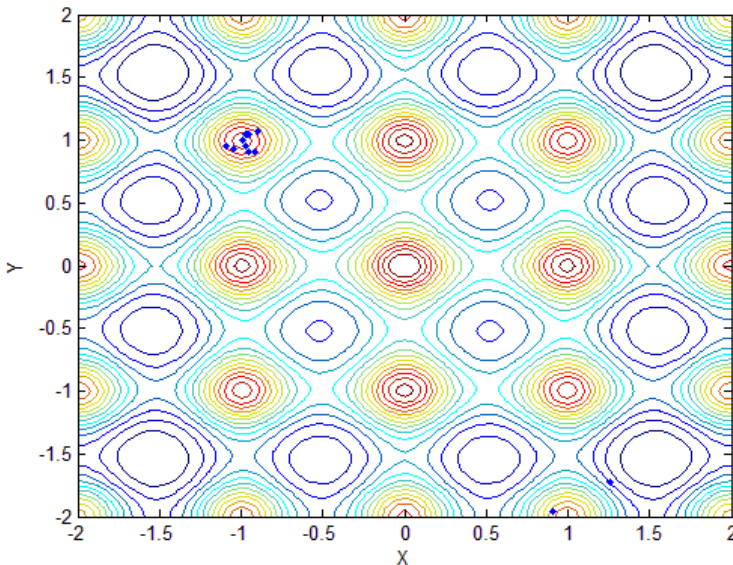

(c) Locations of fireflies for $f_{2}$ with standard method

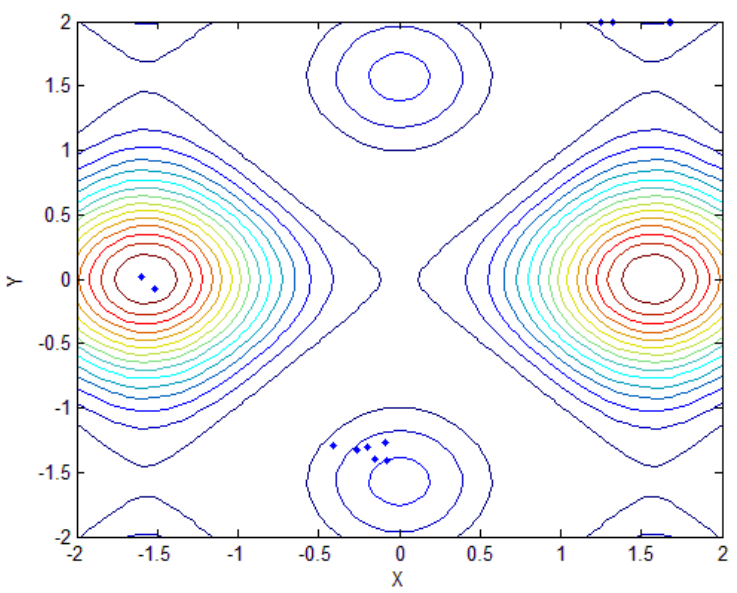

(e) Locations of fireflies for $f_{3}$ with standard method

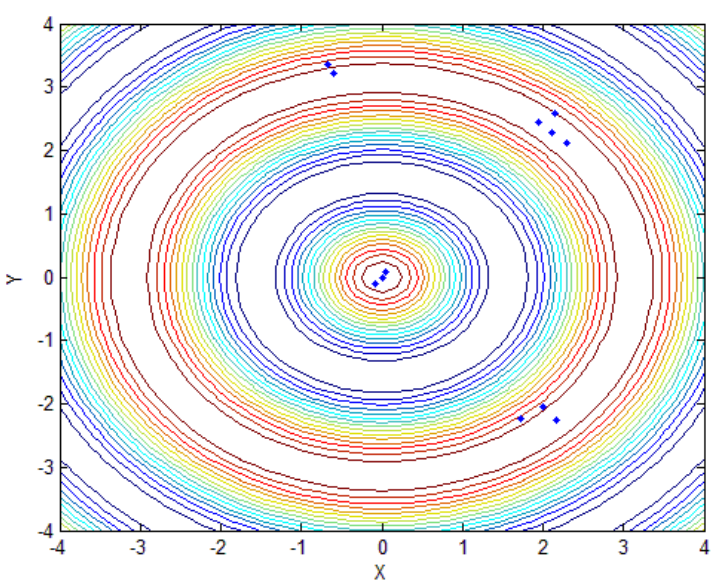

(g) Locations of fireflies for $f_{4}$ with standard meth

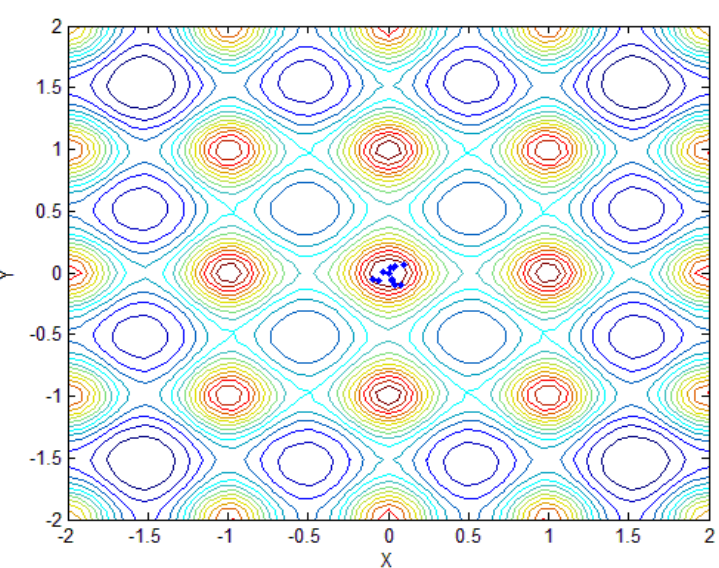

(d) Locations of fireflies for $f_{2}$ with proposed method

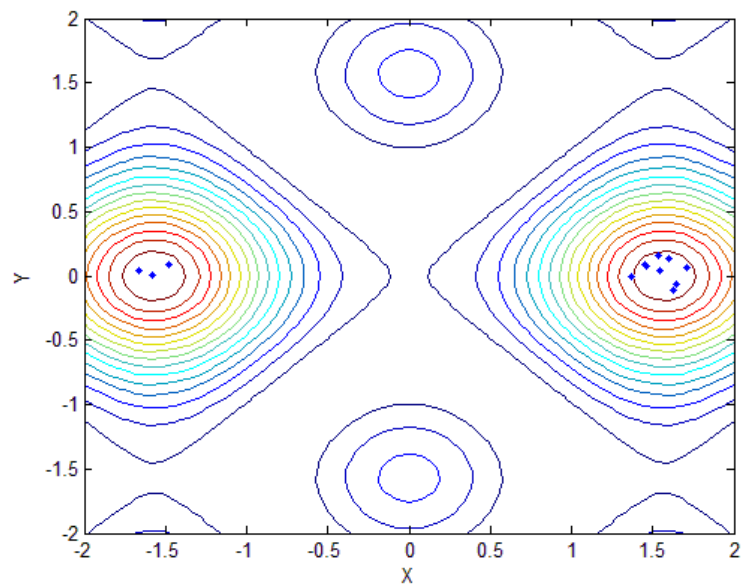

(f) Locations of fireflies for $f_{3}$ with proposed method

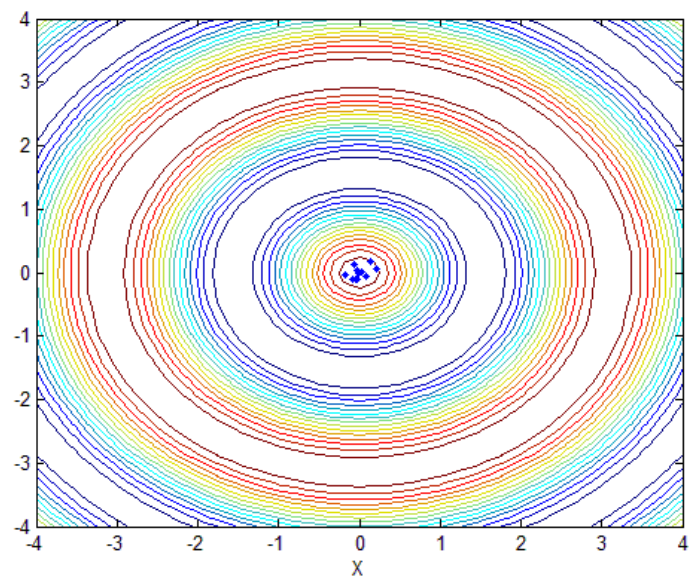

(h) Locations of fireflies for $f_{4}$ with proposed method 


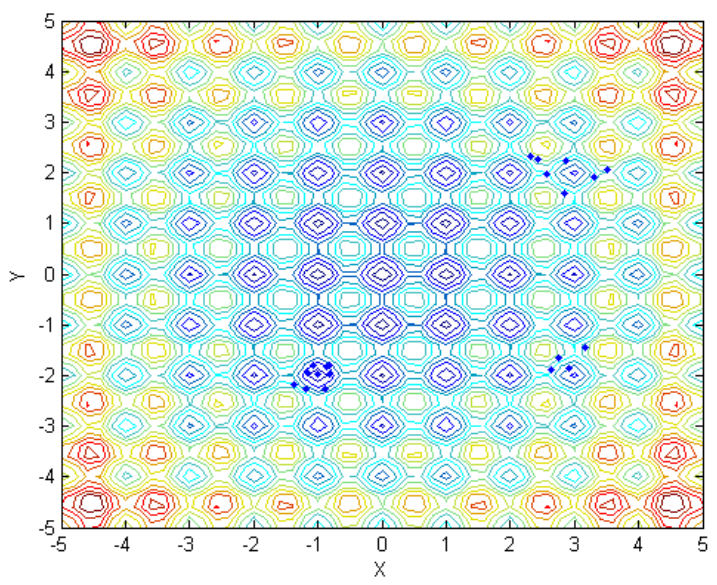

(i) Locations of fireflies for $f_{5}$ with standard method

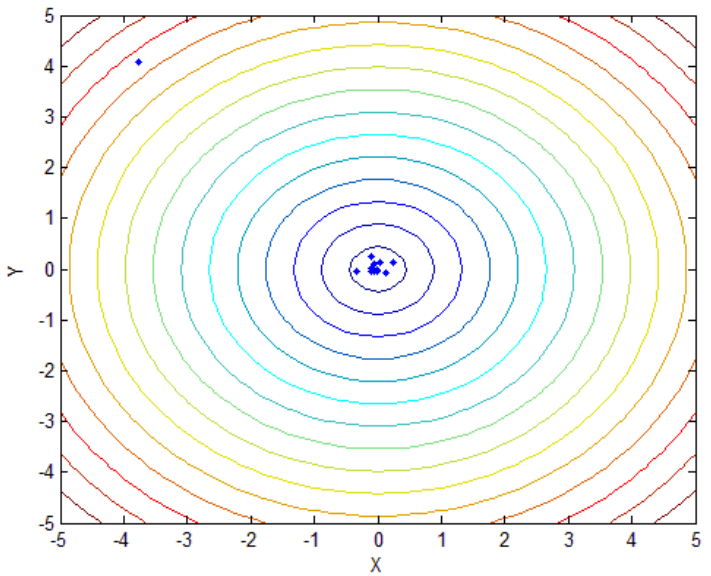

(k) Locations of fireflies for $f_{6}$ with standard method

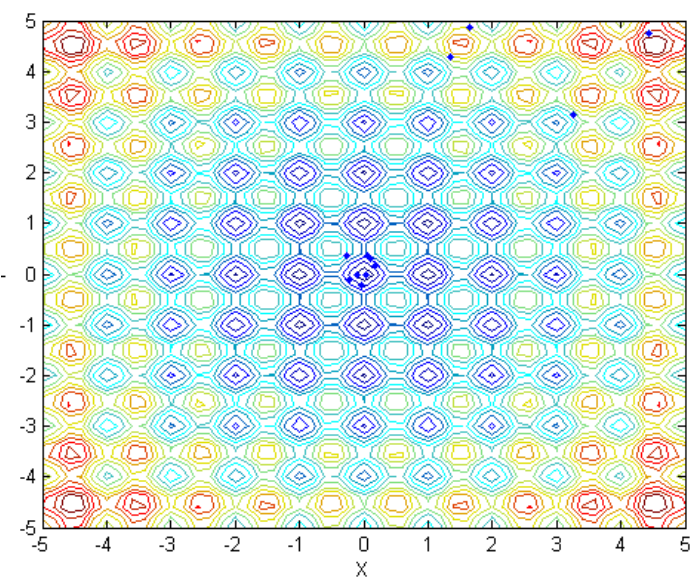

(j) Locations of fireflies for $f_{5}$ with proposed method

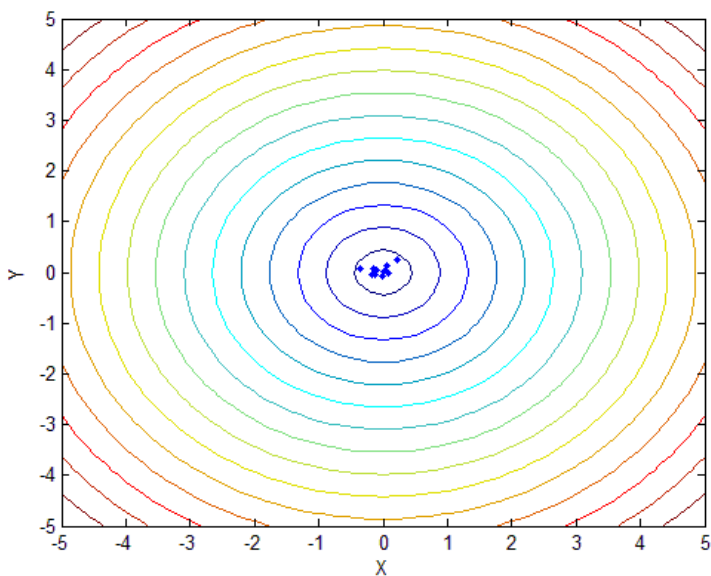

(1) Locations of fireflies for $f_{6}$ with proposed method

Fig.2. Locations of fireflies for $f_{1} \sim f_{6}$ with standard method and proposed method

Table 1. Global max/min value for different functions with standard and proposed method

\begin{tabular}{|c|c|c|c|}
\hline Functions & Methods & Global Max coordinate & $\begin{array}{c}\text { Global Max/ Min } \\
\text { Value }\end{array}$ \\
\hline \multirow{2}{*}{$f_{1}$} & $\begin{array}{c}\text { Standard } \\
\text { method }\end{array}$ & $\begin{array}{c}(-0.0060,0.0109),(0.0580,- \\
4.0509)\end{array}$ & $1.9997,1.9881$ \\
\hline & $\begin{array}{c}\text { Proposed } \\
\text { method }\end{array}$ & $\begin{array}{c}(0.0778,0.0920),(0.0051,- \\
3.9995) \\
\end{array}$ & $1.9712,1.9999$ \\
\hline \multirow{2}{*}{$f_{2}$} & $\begin{array}{c}\text { Standard } \\
\text { method }\end{array}$ & $(-0.9920,0.9978)$ & 0.7048 \\
\hline & $\begin{array}{c}\text { Proposed } \\
\text { method }\end{array}$ & $(0.0152,0.0016)$ & 0.9991 \\
\hline \multirow{2}{*}{$f_{3}$} & $\begin{array}{c}\text { Standard } \\
\text { method }\end{array}$ & $(-1.5955,0.0141)$ & 2.1338 \\
\hline & $\begin{array}{c}\text { Proposed } \\
\text { method }\end{array}$ & $\begin{array}{c}(1.5464,0.0460),(- \\
1.5782,0.0051)\end{array}$ & $2.1303,2.1352$ \\
\hline \multirow{2}{*}{$f_{4}$} & $\begin{array}{c}\text { Standard } \\
\text { method }\end{array}$ & $(0.0157,-0.0030)$ & 0.9997 \\
\hline & $\begin{array}{c}\text { Proposed } \\
\text { method }\end{array}$ & $(-0.0081,-0.0043)$ & 0.9999 \\
\hline \multirow{2}{*}{$f_{5}$} & $\begin{array}{c}\text { Standard } \\
\text { method }\end{array}$ & $(-1.0125,-1.9803)$ & 5.0538 \\
\hline & $\begin{array}{c}\text { Proposed } \\
\text { method }\end{array}$ & $(0.0262,-0.0073)$ & 0.1466 \\
\hline \multirow{2}{*}{$f_{6}$} & $\begin{array}{c}\text { Standard } \\
\text { method }\end{array}$ & $(-0.0027,-0.0094)$ & 0.0098 \\
\hline & $\begin{array}{c}\text { Proposed } \\
\text { method }\end{array}$ & $(0.0011,0.0006)$ & 0.0012 \\
\hline
\end{tabular}




\section{ACKNOWLEDGMENT}

This work was supported by the National Natural Science Foundation of China (61572416), Shenzhen significant strategy layout project (JCYJ20160429112213 821), and Hunan province Natural science Zhuzhou United foundation (2016JJ5033). HuNan University of Science and Technology Doctor Startup Fund Grant No.E51664, Hunan Provincial Department of Education Science Research Project Grant No.16C0639.

\section{REFERENCES}

[1] Xin-She Yang, Chapter 8 - Firefly Algorithms, In NatureInspired Optimization Algorithms, Elsevier, Oxford, 2014, Pages 111-127.

[2] Heba F. Eid, "Performance Improvement of Plant Identification Model based on PSO Segmentation", International Journal of Intelligent Systems and Applications (IJISA), Vol.8, No.2, pp.53-58, 2016. DOI: 10.5815/ijisa.2016.02.07

[3] Yu, W., et al. (2016). "The Faults Diagnostic Analysis for Analog Circuit Based on FA-TM-ELM." Journal of Electronic Testing: 1-7.

[4] D Jinil Persis, T Paul Robert, "Reliable Mobile Ad-Hoc Network Routing Using Fir efly Algorithm", International Journal of Intelligent Systems and Applications (IJISA), Vol.8, No.5, $\quad$ pp.10-18, 2016. DOI:10.5815/ijisa.2016.05.02.

[5] Y.-C. Chang, An outstanding method for saving energyoptimal chiller operation, IEEE Transactions on Energy Conversion 21 (2006) 527-532.

[6] Y.-C. Chang, T.-S. Chan, W.-S. Lee, Economic dispatch of chiller plant by gradient method for saving energy, Applied Energy 87 (2010) 1096-1101.

[7] Surafel Luleseged Tilahun, Hong Choon Ong, Modified firefly algorithm, Journal of Applied Mathematics 2012(2012). doi: http://dx.doi.org/10.1155/2012/467631.

[8] Leandro dos Santos Coelho, Viviana Cocco Mariani, Improved firefly algorithm approach applied to chiller loading for energy conservation, Energy and Buildings, Volume 59, April 2013, Pages 273-278.

[9] Adil Baykasoğlu, Fehmi Burcin Ozsoydan, An improved firefly algorithm for solving dynamic multidimensional knapsack problems, Expert Systems with Applications, Volume 41, Issue 8, 15 June 2014, Pages 3712-3725

[10] Masoud Farhoodnea, Azah Mohamed, Hussain Shareef, Hadi Zayandehroodi, Optimum placement of active power conditioner in distribution systems using improved discrete firefly algorithm for power quality enhancement, Applied Soft Computing, Volume 23, October 2014, Pages 249-258.

[11] M. Gnana Sundari, M. Rajaram, Sujatha Balaraman, Application of improved firefly algorithm for programmed PWM in multilevel inverter with adjustable DC sources, Applied Soft Computing, Volume 41, April 2016, Pages 169-179

[12] M. Gnana Sundari, M. Rajaram, Sujatha Balaraman, Application of improved firefly algorithm for programmed PWM in multilevel inverter with adjustable DC sources, Applied Soft Computing, Volume 41, April 2016, Pages 169-179.

[13] Xin-She Yang, Chapter 2 - Analysis of Algorithms, In Nature-Inspired Optimization Algorithms, Elsevier, Oxford, 2014, Pages 23-44.
[14] Dillip Kumar Mohanty, Application of firefly algorithm for design optimization of a shell and tube heat exchanger from economic point of view, International Journal of Thermal Sciences, Volume 102, April 2016, Pages 228238.

[15] Xin-She Yang, Seyyed Soheil Sadat Hosseini, Amir Hossein Gandomi, Firefly Algorithm for solving nonconvex economic dispatch problems with valve loading effect, Applied Soft Computing, Volume 12, Issue 3, March 2012, Pages 1180-1186.

\section{Authors' Profiles}

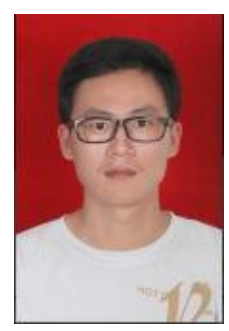

Yong-Bo Sui was born in Shandong, on April 2, 1990. He received the B.E. degree in electrical engineering from Xiangtan University, Xiangtan, Hunan, in 2014. He is currently working toward the M.S. degree at Xiangtan University, Xiangtan, Hunan. His research interest include intelligent algorithm and faults diagnostic analysis.

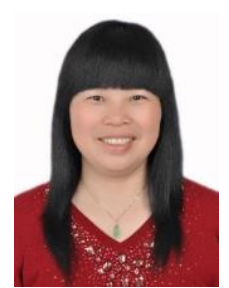

Ling-Zhi Yi was born on March 27, 1966 in Hunan in China. She graduated from the Xiangtan University in 1986. And after then she worked in Xiangtan University, act as the leader of the electricity-electronics committee of Xiangtan University. She is elected as a core young teacher of Hunan province in 1999, and as the person for 121 project of Hunan province in 2007. Up to today, she has published 100 papers in some famous magazines with 4 patents for invention and 18 software copyrights, 51 papers cited by SCI and EI index included. She is now an expert in National Energy Conservation Center, senior member of Chinese Association of Automation and executive director in Institute of Automation of Hunan

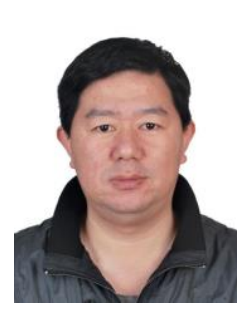

Wen-Xin Yu received the B.Sc. degree in applied mathematics from Hebei Normal University, Shijiazhuang, China, in 2005, and the M.S. degree in wavelet analysis from Changsha University of Science \& Technology, Changsha, in 2008. And Ph.D. degree in electrical engineering from Hunan University. Changsha, in 2015. He joined the Hunan University of Science and Technology, where he currently is lecturer of School of Information and Electrical Engineering. His interests include the research of Fault diagnosis, signal processing, and wavelet analysis and its application.

Manuscript received December 17, 2016; revised December 30, 2016; accepted January 17, 2017.

How to cite this paper: Yongbo Sui, Lingzhi Yi, Wenxin $\mathrm{Yu}$,"A Novel and Improved Firefly Algorithm Based on Twoorder Oscillation", International Journal of Intelligent Systems and Applications(IJISA), Vol.9, No.5, pp.19-26, 2017. DOI: 10.5815/ijisa.2017.05.03 